\title{
ZINC-OXIDE NANOPARTICLES AS FACILE CATALYST FOR RAPID SYNTHESIS OF 5-METHYL-4-(2-(3-METHYL-4- NITROISOXAZOL-5-YL)-1-ARYLETHYL)-1H-PYRAZOL- 3-OLS IN AQUEOUS MEDIUM
}

\author{
P. Usharani ${ }^{1}$ and N. Madhavi* \\ ${ }^{1}$ Department of Chemistry, Acharya Nagarjuna University, Guntur, \\ "PG Department of Chemistry, JKC College, Guntur, Andhra Pradesh, India \\ *E-mail: madhavijkcchempg@gmail.com
}

\begin{abstract}
A Rapid and convenient protocol has been explored for the preparation of diversely functionalized pyrazol-3-ol compounds using zinc oxide nanoparticles as a heterogeneous catalyst. This protocol affords simple to operate, environmentally benign, mild and broadly applicable to synthesize a series of pyrazole-3-ols.

Keywords: Heterogeneous Catalysis, Zinc-oxide Nanoparticles, Multi-component Reaction, 3-Methyl-4-nitro-5alkenylisoxazoles, Pyrazole.
\end{abstract}

CRASĀYAN. All rights reserved

\section{INTRODUCTION}

Heterogeneous catalysis has significant advantages in industrial and academic laboratories owing to its environmental and economical applications. ${ }^{1-5}$ Remarkably, heterogeneous nanoparticles having transition metal are very useful in catalysis due to its cost effective and recyclable nature. The zinc oxide nanoparticles (ZnO NPs) are highly significant transition metal containing nanoparticles because of it's easy to carry, disposal character and non harmful nature. ${ }^{6-10}$ The current literature survey reveals that zinc oxide nanoparticles as heterogeneous catalysts are substantially important in catalysis. ${ }^{11}$ Therefore the development of novel zinc oxide nanoparticles catalysed methods are highly desirable in organic synthesis.

Multicomponent reactions are highly important in synthetic organic chemistry as they offer quick formation of compounds in a single step via multiple bond formation. ${ }^{12-17}$ From green chemistry principles the improvement of new multicomponent reactions in aqueous medium for the preparation of privileged medicinally significant scaffolds are highly useful in drug discovery. ${ }^{18-27}$ Furthermore, pyrazole containing compounds are ubiquitous in several natural products, active pharmaceuticals and agrochemical industries. Remarkably, pyrazoles and their analogues act as antimicrobials and oncology drugs. ${ }^{28-30}$ Specially, several significant commercial drugs having pyrazole framework, for instance celecoxib, ${ }^{31}$ lonazolac $^{32}$ and rimonabant. ${ }^{33}$ Synthesis of such pyrazole analogues using heterogeneous catalyst $\mathrm{ZnO}$ nanoparticles in aqueous medium is always highly significant.

Recently, many protocols have been explored for the preparation of pyrazole derivatives via Michael addition of 2-methylpyrazol-5-one to $\alpha, \beta$-unsaturated carbonyls ${ }^{34-38}$ and $\beta$-nitrostyrenes. ${ }^{39-40}$ Recently, Meshram et. al reported multicomponent reaction to synthesize the pyrazole and isoxazole combined molecules. ${ }^{41}$ However, in this method they used toxic and non reusable piperdine as a catalyst at $80^{\circ} \mathrm{C}$. From literature review, it is identified that no method is reported for the preparation of pyrazolone functionalized isoxazoles by one pot, three component reaction of hydrazine hydrate, ethylacetoacetate and 3-methyl-4-nitro-5-alkenylisoxazole using non toxic zinc oxide nanoparticles as a heterogeneous

Rasayan J. Chem., 12(4), 2023-2029(2019)

http://dx.doi.org/10.31788/RJC.2019.1245468

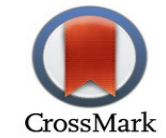




\section{RASĀYAN J. Chem.}

Vol. 12 | No. 4 |2023 - 2029| October - December | 2019

catalyst. Hence an attempt is made to report a simple, convenient and mild protocol for the preparation of 5-methyl-4-(2-(3-methyl-4-nitroisoxazol-5-yl)-1-arylethyl)-1H-pyrazol-3-ol.

\section{Materials and Methods}

\section{EXPERIMENTAL}

All chemicals and solvents are purchased from Avra Synthesis Pvt. Ltd. and Sigma Aldrich company, which are utilized without purification as received. Melting points are taken in a capillary and are uncorrected. ${ }^{1} \mathrm{H}-\mathrm{NMR}$ and ${ }^{13} \mathrm{C}-\mathrm{NMR}$ are recorded on a Bucker 300 instrument and IR spectrum is recorded in $\mathrm{KBr}$ pellets on a Nicolet impact.

\section{General Reaction Procedure for Preparation of Pyrazol-3-ol Derivatives}

In a typical experiment the ethylacetoacetate 1 (1 mmol), hydrazine hydrate $\mathbf{2}(1 \mathrm{mmol}), 3$-methyl-4-nitro5-alkenylisoxazoles $3(1 \mathrm{mmol}), \mathrm{ZnO}(10 \mathrm{~mol} \%)$ and water $(3 \mathrm{~mL})$ are taken in a $25 \mathrm{~mL}$ round-bottomed flask and stirred at $30^{\circ} \mathrm{C}$ for 15 mins. After the end of reaction (observed via TLC),it is filtered using sintered glass funnel, and the resulting solid is washed with hot $\mathrm{H}_{2} \mathrm{O}$ and $\mathrm{Et}_{2} \mathrm{O}$ and recrystallized in $\mathrm{C}_{2} \mathrm{H}_{5} \mathrm{OH}$ to achieve pure compound.

\section{General Reaction Procedure for Synthesis of Zinc-Oxide (Zno) Nanoparticles}

$\mathrm{ZnO}$ nanoparticles are prepared via precipitation protocol as reported in literature, ${ }^{42}$ and the prepared zinc-oxide nanoparticles are analyzed by powder-XRD, FT-IR and SEM.

\section{Analytical Discussion}

\section{Compound 4a (Yellow Solid)}

Yield $89 \%$; MP: $189-191{ }^{\circ} \mathrm{C}, \mathrm{IR}\left(\mathrm{KBr}, \mathrm{cm}^{-1}\right): 3411(\mathrm{R}-\mathrm{OH}), 2926\left(\mathrm{R}_{-} \mathrm{CH}_{3}\right), 2570(=\mathrm{C}-\mathrm{H}), 1602(\mathrm{C}=\mathrm{C}$ arom), 1517-1418 (N-O); ${ }^{1} \mathrm{H}-\mathrm{NMR}\left(300 \mathrm{MHz}, \mathrm{CDCl}_{3}+\mathrm{DMSO}_{6}\right) ; 7.48(\mathrm{~s}, 1 \mathrm{H}), 7.37(\mathrm{~d}, J=7.3 \mathrm{~Hz}, 2 \mathrm{H})$, 7.13-7.27 (m, 3H), $4.41(\mathrm{t}, J=8.1 \mathrm{~Hz}, 1 \mathrm{H}), 4.01(\mathrm{~d}, J=8.1 \mathrm{~Hz}, 2 \mathrm{H}), 2.47(\mathrm{~s}, 3 \mathrm{H}), 2.07(\mathrm{~s}, 3 \mathrm{H}) ;{ }^{13} \mathrm{C} \mathrm{NMR}$ (75 MHz, $\mathrm{CDCl}_{3}+$ DMSO-d $_{6}$ ): 173.42, 160.11, 154.65, 142.57, 137.28, 127.85, 126.72, 126.00, 101.08, 37.38, 32.02, 10.95, 9.67; ESI-MS: m/z: $329(\mathrm{M}+\mathrm{H})^{+}$.

\section{Compound 4c (Yellow Solid)}

Yield $97 \%$; MP: 225-227 ${ }^{\circ} \mathrm{C}$; IR ( $\left.\mathrm{KBr}, \mathrm{cm}^{-1}\right)$ : 3405 (R-OH), $2934\left(\mathrm{R}^{-} \mathrm{CH}_{3}\right), 1603$ (C=C arom), 1514-1417 $(\mathrm{N}-\mathrm{O}) ;{ }^{1} \mathrm{H}-\mathrm{NMR}\left(300 \mathrm{MHz}, \mathrm{CDCl}_{3}+\mathrm{DMSO}_{-} \mathrm{d}_{6}\right): 7.88(\mathrm{~s}, 1 \mathrm{H}), 7.26(\mathrm{~d}, J=8.6 \mathrm{~Hz}, 2 \mathrm{H}), 6.76(\mathrm{~d}, J=8.1$ $\mathrm{Hz}, 2 \mathrm{H}), 4.31(\mathrm{t}, J=8.1 \mathrm{~Hz}, 1 \mathrm{H}), 3.93(\mathrm{~d}, J=8.1 \mathrm{~Hz}, 2 \mathrm{H}), 3.74(\mathrm{~s}, 3 \mathrm{H}), 2.45(\mathrm{~s}, 3 \mathrm{H}), 2.03(\mathrm{~s}, 3 \mathrm{H}) ;{ }^{13} \mathrm{C}$ NMR (75 MHz, $\mathrm{CDCl}_{3}+\mathrm{DMSO}_{6}$ ) 172.41, 158.05, 156.19, 153.34, 135.35, 133.90, 128.30, 126.54, 111.98, 100.21, 53.34, 35.35, 30.90, 9.69, 8.42; ESI-MS: m/z: $359(\mathrm{M}+\mathrm{H})^{+}$.

\section{Compound 4e (Colourless Solid)}

Yield $92 \%$; MP: 204-206 ${ }^{\circ} \mathrm{C}$; IR $\left(\mathrm{KBr}, \mathrm{cm}^{-1}\right) 3319(\mathrm{R}-\mathrm{OH}), 2928\left(\mathrm{R}_{-} \mathrm{CH}_{3}\right), 2604(=\mathrm{C}-\mathrm{H}), 1605(\mathrm{C}=\mathrm{C}$ arom), 1520-1491 (N-O); ${ }^{1} \mathrm{H}-\mathrm{NMR}\left(300 \mathrm{MHz}, \mathrm{CDCl}_{3}+\mathrm{DMSO}_{6}\right) ; 7.60$ (s, 1H), 7.34 (d, J = 8.4 Hz, 2H), $7.19(\mathrm{~d}, J=8.4 \mathrm{~Hz}, 2 \mathrm{H}), 4.37(\mathrm{t}, J=7.93 \mathrm{~Hz}, 1 \mathrm{H}), 3.98(\mathrm{~d}, J=8.1 \mathrm{~Hz}, 2 \mathrm{H}), 2.47(\mathrm{~s}, 3 \mathrm{H}), 2.07(\mathrm{~s}, 3 \mathrm{H}) ;{ }^{13} \mathrm{C}$ NMR (125 MHz, $\mathrm{CDCl}_{3}+\mathrm{DMSO}_{6}$ ) 172.61, 159.31, 154.14, 140.93, 136.66, 130.88, 129.11, 127.84, 127.37, 100.16, 36.36, 31.40, 10.46, 9.13; ESI-MS: m/z: $379(\mathrm{M}+\mathrm{H})^{+}$.

\section{Compound 4k (Colourless Solid)}

Yield $85 \%$; MP: $177-179{ }^{\circ} \mathrm{C}$; IR $\left(\mathrm{KBr}, \mathrm{cm}^{-1}\right) 3346(\mathrm{R}-\mathrm{OH}), 2925\left(\mathrm{R}-\mathrm{CH}_{3}\right), 2624(=\mathrm{C}-\mathrm{H}), 1602(\mathrm{C}=\mathrm{C}$ arom), 1520-1422 (N-O); ${ }^{1} \mathrm{H}-\mathrm{NMR}\left(300 \mathrm{MHz}, \mathrm{CDCl}_{3}+\mathrm{DMSO}_{\mathrm{d}}\right)$ : $7.54(\mathrm{~s}, 1 \mathrm{H}), 7.30(\mathrm{~s}, 1 \mathrm{H}), 6.26(\mathrm{t}, J=$ $3.0 \mathrm{~Hz}, 1 \mathrm{H}), 6.09(\mathrm{~d}, J=3.2 \mathrm{~Hz}, 1 \mathrm{H}), 4.54(\mathrm{t}, J=7.7 \mathrm{~Hz}, 1 \mathrm{H}), 3.95(\mathrm{dd}, J=7.1,14.9 \mathrm{~Hz}, 1 \mathrm{H}), 3.84(\mathrm{dd}, J$ $=8.6,14.9 \mathrm{~Hz}, 1 \mathrm{H}), 2.50(\mathrm{~s}, 3 \mathrm{H}), 2.09(\mathrm{~s}, 3 \mathrm{H}) ;{ }^{13} \mathrm{C} \mathrm{NMR}\left(75 \mathrm{MHz}, \mathrm{CDCl}_{3}+\mathrm{DMSO}_{\mathrm{d}}\right)$ 171.84, 158.58, 153.86, 139.96, 136.47, 108.94, 104.31, 97.70, 29.86, 29.57, 10.11, 8.83; ESI-MS: m/z: $319(\mathrm{M}+\mathrm{H})^{+}$.

\section{RESULTS AND DISCUSSION}

The synthesized $\mathrm{ZnO}$ nanoparticles are characterized by powder-XRD, FT-IR and SEM as shown in Figures-1, 2 and 3. 
RASĀYAN J. Chem.

Vol. 12 | No. 4 |2023 - 2029| October - December | 2019

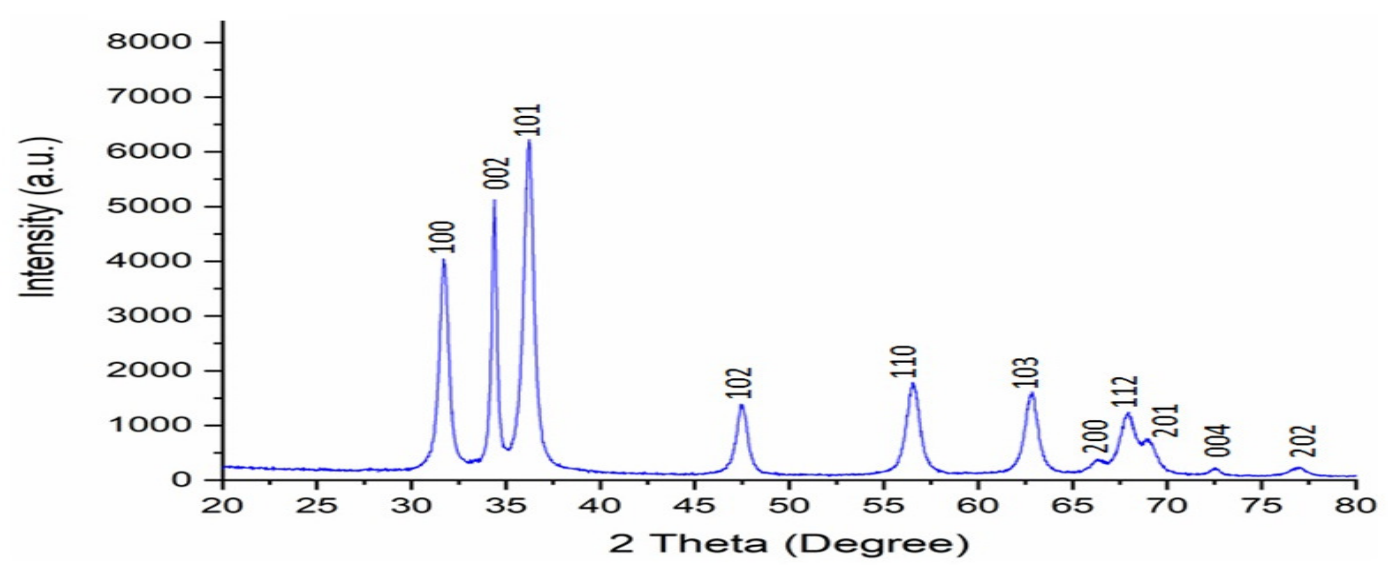

Fig.-1: Powder XRD pattern of ZnO Nanoparticles

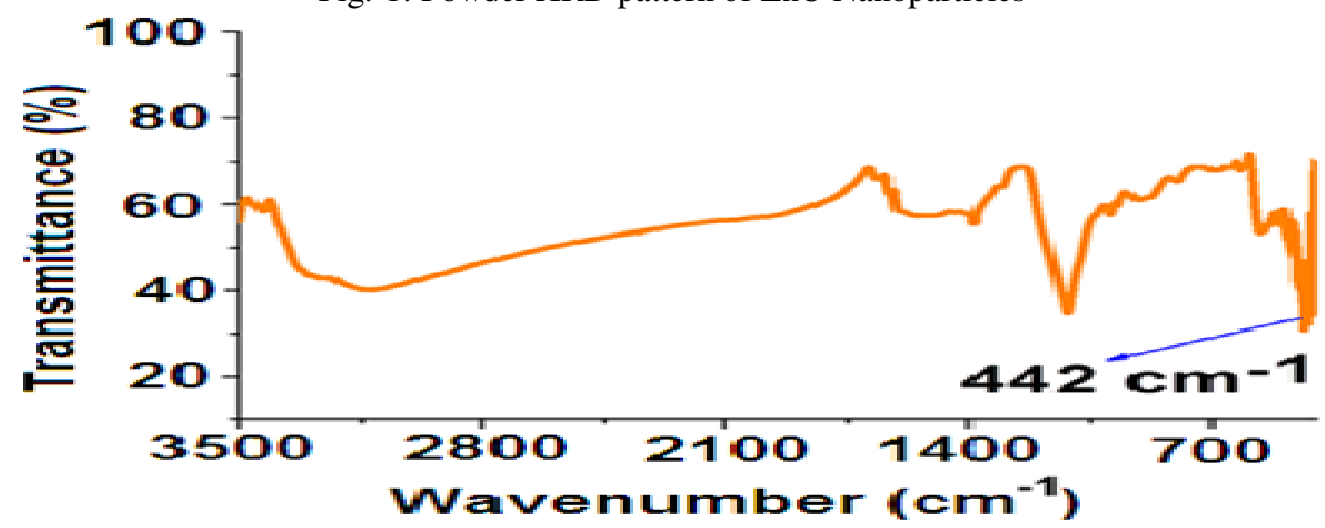

Fig.-2: FT-IR Spectra of ZnO Nanoparticles

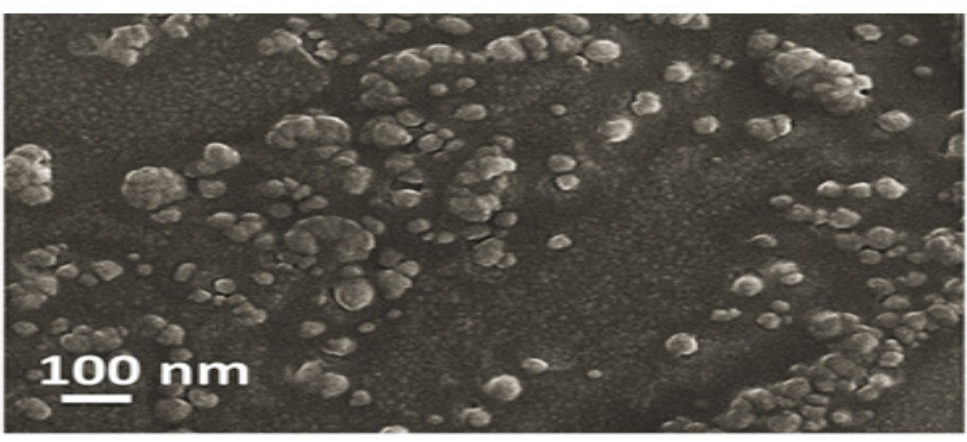

Fig.-3: SEM Image of ZnO Nanoparticles.<smiles>CCOC(=O)CC(C)=O</smiles>

2<smiles>Cc1noc(/C=C/c2ccccc2)c1[N+](=O)[O-]</smiles>

$3 a$<smiles>Cc1noc(CC(c2ccccc2)c2c(O)n[nH]c2C)c1[N+](=O)[O-]</smiles>

$4 a$

Scheme-1: Optimization Studies

Initially, ethyl acetoacetate (1), hydrazine hydrate (2) and (E)-3-methyl-4-nitro-5-styrylisoxazole (3a) as model substrates are employed to test the desired three component reaction in aqueous medium (Scheme1).The reaction has carried a reaction using $1.0 \mathrm{mmol}$ of $\mathbf{1}, 1.0 \mathrm{mmol}$ of $\mathbf{2}, 1.0 \mathrm{mmol}$ of $\mathbf{3 a}, 5 \mathrm{~mol} \%$ of 
RASĀYAN J. Chem.

Vol. 12 | No. 4 |2023 - 2029| October - December | 2019

$\mathrm{ZnO}$ and $\mathrm{H}_{2} \mathrm{O} 3 \mathrm{~mL}$ at room temperature. After $60 \mathrm{mins}$, it is found that $65 \%$ of the product is formed (Table-1, entry 1). Later, it is carried reaction with $1.0 \mathrm{mmol}$ of $\mathbf{1}, 1.0 \mathrm{mmol}$ of $2,1.0 \mathrm{mmol}$ of $\mathbf{3 a}$ and water $3 \mathrm{~mL}$ with diverse mol percentage i.e 10, 15, and 25 of $\mathrm{ZnO}$ as shown in above Table 1(entries 2-4). Reaction time also optimized and it is observed that the increase in yield with increase in mol percentage of $\mathrm{ZnO}$ (Table 1, entry 5 and 6). Finally, it is examined by using $1.0 \mathrm{mmol}$ of $\mathbf{1}, 1.0 \mathrm{mmol}$ of $\mathbf{2}, 1.0 \mathrm{mmol}$ of $3 \mathrm{a}, \mathrm{ZnO}(10 \mathrm{~mol} \%)$ and water $3 \mathrm{~mL}$ at $35{ }^{\circ} \mathrm{C}$ for $15 \mathrm{mins}$ and the required compound is formed by 95 $\%$ of yield (Table-1, entry 6). The structure of required compound is proved by using spectral analysis $\left({ }^{1} \mathrm{H},{ }^{13} \mathrm{C}\right.$, IR and mass spectral data).

\begin{tabular}{c|c|c|c|c}
\multicolumn{5}{|c}{ Table-1: Optimization Studies } \\
\hline S. No. & Zincoxide $(\mathrm{ZnO})(\mathrm{mol} \%)$ & Reaction medium & ${\text { Time }(\mathrm{min})^{\mathrm{b}}}^{\left.\text {Yield }^{\mathrm{a}} \%\right)^{\mathrm{c}}}$ \\
\hline 1 & 5 & $\mathrm{H}_{2} \mathrm{O}$ & 60 & 65 \\
\hline 2 & 10 & $\mathrm{H}_{2} \mathrm{O}$ & 60 & 95 \\
\hline 3 & 15 & $\mathrm{H}_{2} \mathrm{O}$ & 60 & 94 \\
\hline 4 & 25 & $\mathrm{H}_{2} \mathrm{O}$ & 60 & 95 \\
\hline 5 & 10 & $\mathrm{H}_{2} \mathrm{O}$ & 30 & 95 \\
\hline $\mathbf{6}$ & $\mathbf{1 0}$ & $\mathbf{H}_{2} \mathbf{O}$ & $\mathbf{1 5}$ & $\mathbf{9 5}$ \\
\hline 7 & 10 & $\mathrm{EtOH}$ & 60 & 50 \\
\hline 8 & 10 & $\mathrm{MeOH}$ & 60 & 65 \\
\hline
\end{tabular}

a All reactions are carried out with 1 (1 mmol), 2 (1 mmol), 3a $(1 \mathrm{mmol})$ and $3 \mathrm{~mL} \mathrm{H}_{2} \mathrm{O}$.

${ }^{b}$ Reaction time in minutes. ${ }^{\mathrm{c}}$ Isolated yields of the Product.

From the observed reaction conditions, it is examined that the substrate scope of the present eco-friendly protocol. The protocol is proceeding well with various 3-methyl-4-nitro-5-alkenylisoxazoles as depicted in Table-2. The reaction gave good yields with 3-methyl-4-nitro-5-alkenylisoxazoles having electron withdrawing as well as donating groups on phenyl ring. The reaction is also carried out by using heterocyclic styryl isoxazoles $3 \mathrm{k}$ and 31 fruitfully as substrates.

Based on the earlier reports and above observations, a suitable pathway is proposed as shown in Scheme3. Initially, ethylacetoacetate $\mathbf{1}$ and hydrazine hydrate 2 reacts to form 3-methyl-pyrazole-5-one (A). $\mathrm{ZnO}$ helps to form an enol intermediate from the 3-methyl-pyrazole-5-one (B). Which consecutively undergoes addition with (E)-3-methyl-4-nitro-5-styrylisoxazole (3) followed by rearrangement to form the corresponding compound $\mathbf{4}$ as shown in Scheme-2.

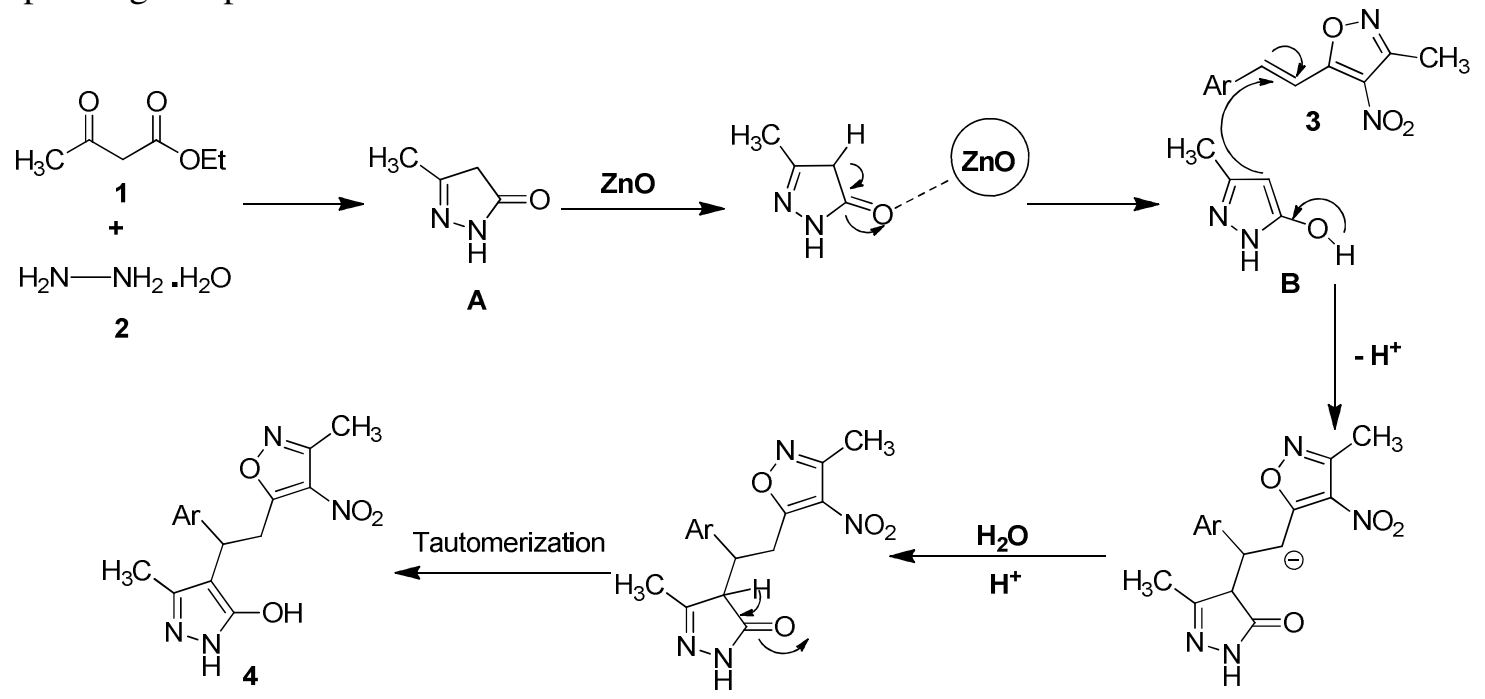

Scheme-2: Plausible Mechanism

\section{CONCLUSION}

An efficient and eco-friendly protocol has been developed for the preparation of diverse pyrazole derivatives via cyclization followed by Michael addition in water using $\mathrm{ZnO}$ as a heterogeneous catalyst. The method has excellent tolerance for diverse reactants. It is important to mention that the protocol not 
RASĀYAN J. Chem.

Vol. 12 | No. 4 |2023 - 2029| October - December | 2019

require organic reaction medium. The pure form of product is isolated without using column chromatography which is an added advantage of this green protocol.

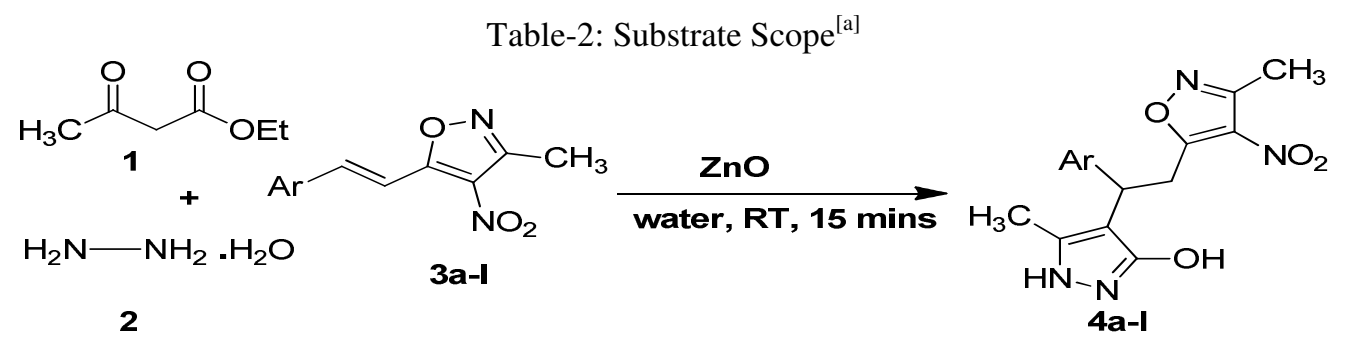

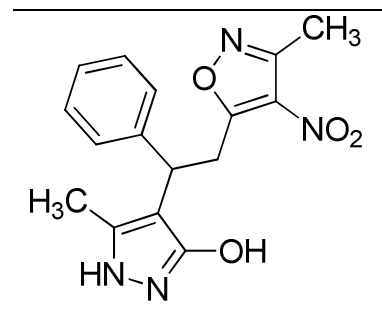

$4 a, 95 \%$ b<smiles>Cc1n[nH]c(O)c1C(Cc1onc(C)c1[N+](=O)[O-])c1ccc(Cl)cc1</smiles>

$4 e, 92 \%^{b}$

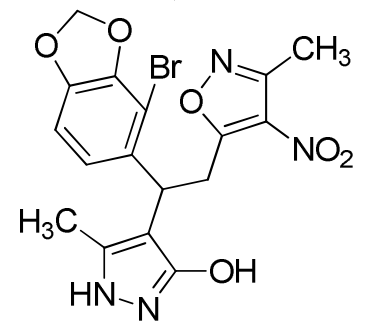

$4 i, 92 \%$<smiles>Cc1ccc(C(Cc2onc(C)c2C)c2c(O)n[nH]c2C)cc1</smiles>

$4 b, 97 \%$<smiles>Cc1n[nH]c(O)c1C(Cc1onc(C)c1[N+](=O)[O-])c1ccc([N+](=O)[O-])cc1</smiles>

4f, $90 \%^{b}$

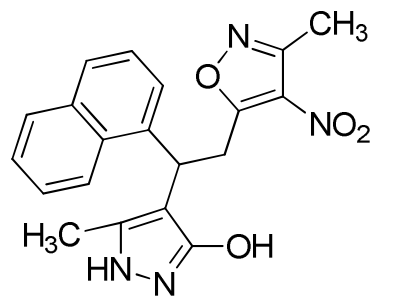

$4 j, 89 \%^{b}$<smiles>COc1ccc(C(Cc2onc(C)c2[N+](=O)[O-])c2c(C)n[nH]c2O)cc1</smiles>

4c, $97 \%$<smiles>COc1ccc(C(Cc2onc(C)c2[N+](=O)[O-])c2c(O)n[nH]c2C)cc1OC</smiles>

$4 \mathrm{~g}, 90 \%$<smiles>Cc1n[nH]c(O)c1C(Cc1onc(C)c1[N+](=O)[O-])c1ccco1</smiles>

$4 k, 85 \%^{b}$

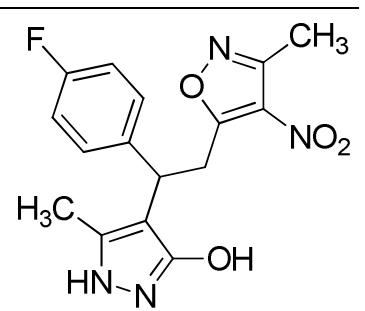

4d, $90 \%$

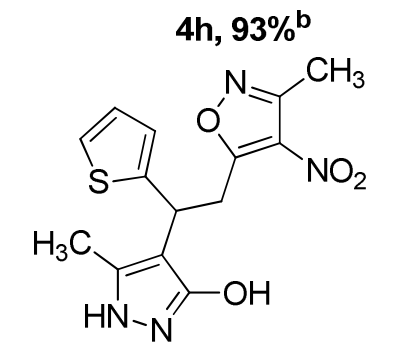

$41,85 \%^{b}$

${ }^{a}$ Reaction conditions: All reactions are performed by $1(1 \mathrm{mmol}), \mathbf{2}(1 \mathrm{mmol}), \mathbf{3 a}(1 \mathrm{mmol}), \mathrm{ZnO}(10$ $\mathrm{mol} \%$ ) and $3 \mathrm{~mL}$ water. ${ }^{\mathrm{b}}$ Yield of the product.

\section{ACKNOWLEDGMENT}

We are thankful to the management, JKC College, Guntur, Andhrapradesh for providing lab facilities.

\section{REFERENCES}

1. M. Moreno-Ma nas, R. Pleixats, Accounts of Chemical Research, 36, 638 (2003), DOI:10.1021/ar020267y

2. D. Astruc, Nanoparticles and Catalysis, Wiley-VCH Verlag GMBH \& Co, 2008.

3. L. Djakovitch, K. Koehler, J.G. Vries, Nanopart. Catal., 65, 303 (2008).

4. J. Durand, E. Teuma and M. G'omez, European Journal of Inorganic Chemistry, 23, 3577 (2008), DOI: 10.1002/ejic.200800569

5. H.H. Kung, Transition Metal Oxides: Surface Chemistry and Catalysis, Elsevier Science, New York, NY, USA, 1989 
RASĀYAN J. Chem.

Vol. 12 | No. 4 |2023 - 2029| October - December | 2019

6. S.M. George, Chem Rev, 95(3), 475(1995), DOI:10.1021/cr00035a001

7. F. Zaera, Chem Soc Rev, 42, 2746(2013), DOI:10.1039/C2CS35261C

8. J.-H. Liu, X. Ma, Y. Xu, H. Tang, S.-T. Yang, Y.-F. Yang, D.-D. kang, H. Wang and Y. Liu, J Toxicol Res, 6(2), 134(2017), DOI: 10.1039/c6tx00370b

9. W. Huang, D. Bai, L. Li, H. Wei, Z. Shi, H. Cheng and Y. Li, J Sol-Gel Sci Technol, 74(3), 718 (2015), DOI:10.1007/s10971-015-3653-0

10. G. Madhumitha, G. Elango and S.M. Roopan, Appl Microbiol Biotechnol, 100(2), 571 (2016), DOI: 10.1007/s00253-015-7108-X.

11. H. Sachdeva and R. Saroj, The Scientific World Journal, 2013, 1 (2013), DOI:10.1155/2013/680671

12. I. Ugi, A. Dömling and W. Horl, Endeavour, 18(3), 115(1994), DOI:10.1016/S01609327(05)80086-9

13. C. Hulme and V. Gore, Curr. Med. Chem. 10(1), 51(2003), DOI:10.2174/0929867033368600

14. J.D. Sunderhaus, C. Dockendorff and S.F. Martin, Org. Lett. 9(21), 4223(2007), DOI:10.1021/ol7018357

15. R.W. Armstrong, A.P. Combs, P.A. Tempest, S.D. Brown and T.A. Keating, Acc. Chem. Res. 29, 123 (1996), DOI:10.1021/ar9502083

16. F.L. Muller, T. Constantieux and J. Rodriguez, J. Am. Chem. Soc. 127(49), 17176(2005), DOI: $10.1021 / \mathrm{ja} 055885 \mathrm{z}$

17. C. Haurena, E.L. Gall, S. Sengmany, T. Martens and M. Troupel, J. Org. Chem. 75, 2645 (2010), DOI: $10.1021 /$ jo1002328

18. L.F. Tietze and M.E. Lieb, Curr. Opin. Chem. Biol. 2, 363(1998), DOI:10.1016/S13675931(98)80010-0

19. S.L. Dax, J.J. McNally and M.A. Youngman, Curr. Med. Chem. 6(3), 255 (1999).

20. B. Willy and T.J.J. Müller, Eur. J. Org. Chem. 4157 (2008), DOI:10.1002/ejoc.200800444

21. M.M. Heravi, B. Baghernejad, H. A. Oskooie and R. Hekmatshoar, Tetrahedron Lett. 49, 6101 (2008), DOI:10.1016/j.tetlet.2008.08.012

22. M. Adib, E. Sheikhi, A. Kavoosi, H.R. Bijanzadeh, Tetrahedron, 66, 9263(2010), DOI: 10.1016/j.tet.2010.09.032

23. A. Dömling, Comb. Chem. High Throughput Screening, 1, 1(1998).

24. N.M. Evdokimov, A.S. Kireev, A.A. Yakovenko, M.Y. Antipin, I.V. Magedov and A. Kornienko, J. Org. Chem. 72(9), 3443(2007), DOI:10.1021/jo070114u

25. K. Kumaravel and G. Vasuki, Curr.Org. Chem. 13(18), 1820(2009), DOI: $10.2174 / 138527209789630514$

26. A. Chanda and V.V. Fokin, Chem. Rev. 109, 725 (2009), DOI: 10.1021/cr800448q

27. D. Tejedor and F. Garcia-Tellado, Chem. Soc.Rev. 36, 484 (2007), DOI: 10.1039/B608164A

28. H. Shiohara, H. Fujikura, N. Fushimi, F. Ito and M. Isaji, PCT Int. Appl. WO., 09, 8893(2002).

29. N. Foloppe, L. M. Fisher, R. Howes, A. Potter, A. G. S. Robertson and A. E. Surgenor, Bioorg. Med. Chem., 14(14), 4792 (2006), DOI: 10.1016/j.bmc.2006.03.021

30. D.A. Barawkar, A. Meru, A. Bandyopadhyay, A. Banerjee, A. M. Deshpande, C. Athare, C. Kodurum, G. Khose, J. Gundu, K. Mahajan, P. Patil, S. R. Kandalkar, S. Niranjan, S. Bhosale, S. De, S. Mukhopadhyay, S. Chaudhary, S. Koul, U. Singh, A. Chugh, V. P. Palle, K. A. Mookhtiar, J. Vacca, P. K. Chakravarty, R. P. Nargund, S. D. Wright, S. Roy, M. P. Graziano, S. B. Singh, D. Cully and T.-Q. Cai, ACS Med. Chem. Lett., 2(12), 919 (2011), DOI: 10.1021/ml2001938

31. T.D. Penning, J. Med. Chem., 40, 1347 (1997), DOI: 10.1021/jm960803q

32. R. Riedel, Arzneimittelforschung., 31, 655 (1981).

33. M. Isidro and F. Cordido, Mini Rev. Med. Chem. 9(6), 664(2009), DOI: 10.2174/138955709788452739

34. H. A. Etman, A. S. El-Ahl and M. A. Metwally, Arch. Pharm. Res., 17, 278(1994), DOI: 10.1007/BF02980461

35. S. Gogoi and C.G. Zhao, Tetrahedron Lett., 50, 2252(2009), DOI: 10.1016/j.tetlet.2009.02.210 


\section{RASĀYAN J. Chem.}

Vol. 12 | No. 4 |2023 - 2029| October - December | 2019

36. A.N. Alba, A. Zea, Valero, G. Calbet, M. T. Font-Bardia, A. Mazzanti, A. Moyano and R. Rios, Eur. J. Org. Chem. 1318 (2011), DOI: 10.1002/ejoc.201001452

37. Z. Wang, Z. Yang, D. Chen, X. Liu, L. Lin and X. Feng, Angew. Chem., 123, 5030 (2011), DOI: 10.1002/anie.201008256

38. H. Mecadon, M.R. Rohman, L. Kharbangar, B.M. Laloo, L. Kharkongor, M. Rajbangshi and B. Myrboh, Tetrahedron Lett., 52, 3228(2011), DOI:10.1016/j.tetlet.2011.04.048

39. H.M. Meshram, N.S. Kumar, N.J. Babu, L.C. Rao and N.N. Rao, Tetrahedron Lett., 54, 5941 (2013), DOI:10.1016/j.tetlet.2013.08.042

40. K.S. Rao, P. Ramesh, R. Trivedi and M.L. Kantam, Tetrahedron Lett., 57, 1227 (2016), DOI: 10.1016/j.tetlet.2016.02.008

41. N. Muthineni, N.S. Kumar, L.C. Rao, V.D. Kumar, S. Misra, L.R. Chowhan and H.M. Meshram, ChemistrySelect. 1, 4197 (2016), DOI:10.1002/slct.201600915

42. R. Suntako, Bull. Mater. Sci. 38, 1033 (2015).

[RJC-5468/2019] 